\title{
Induction of vitiligo after imiquimod treatment of condylomata acuminata
}

Wenfei Li $i^{*}$, Hongyan Xin ${ }^{1,2}$, Lingzhi Ge ${ }^{1,3}$, Haiyan Song ${ }^{1,4}$ and Wang Cao ${ }^{3,5}$

\begin{abstract}
Background: Condylomata acuminata (genital warts) is the most common sexually transmitted disease, and imiquimod is the sole FDA-approved medication for combating this condition. Vitiligo associated with imiquimod treatment of condylomata acuminata is rare.

Case presentation: A 28-year-old male with condylomata acuminata of the penis presented to our clinic. After removing his condylomata acuminata, we advised him to use imiquimod $5 \%$ cream to prevent relapse. When he presented to our clinic again about 12 weeks later, he complained of vitiligo patches on his penis and scrotum. Physical examination showed vitiligo patches involving the glans penis, shaft of the penis, and scrotum, and remaining pigmented areas within the plaques of vitiligo.

A skin biopsy of the dorsal surface of the penis showed a complete absence of melanocytes and melanin granules in the basal layer; the dermis was normal.
\end{abstract}

Conclusion: This is the first report of a case of imiquimod-induced vitiligo diagnosed by histopathological examination. This adverse effect should be considered when dermatologists prescribe this medication.

Keywords: Condylomata acuminata, Imiquimod, Vitiligo

\section{Background}

Condylomata acuminata (genital warts) is the most common sexually transmitted disease. It is caused by human papilloma virus (HPV) infection, which may contribute to cervical cancer [1]. The primary goals of treatment are removal of the visible warts and prevention of recurrence. The many methods in the therapy of condylomata acuminata include cryotherapy, electrodessication, $\mathrm{CO}_{2}$ laser, trichloroacetic acid, podophyllin resin $10 \%-25 \%$, and imiquimod 5\% cream [2,3]. Among them, imiquimod is the sole FDA-approved medication for combating condylomata acuminata. It is also used to treat certain diseases of the skin such as Bowen's disease, common and plantar warts, molluscum contagiosum, herpes simplex, Paget's disease, basal cell carcinoma, and superficial squamous cell carcinoma. Although regarded as a safe drug, mild-to-moderate, local and systemic, adverse effects of imiquimod may occasionally occur [4]. Since vitiligo-like hypopigmentation associated with imiquimod treatment

\footnotetext{
*Correspondence: Iwf888026@163.com

'Department of Dermatology, Qianfoshan Hospital, Shandong University, 16766\# Jing-Shi Road, Jinan 250014, China

Full list of author information is available at the end of the article
}

of condylomata acuminata was first reported by Brown in 2005 , to the best of our knowledge there have been only eight patients with either vitiligo or vitiligo-like hypopigmentation associated with imiquimod treatment of condylomata acuminata described in the literature [5-11]. The clinical features of these patients are listed in Table 1.

Here, we present an unusual case of imiquimodinduced vitiligo in a 28 -year-old male, whose diagnosis was made using clinical and histopathological methods.

\section{Case presentation}

A 28-year-old Chinese male presented to our clinic with a 3-year history of condylomata acuminata of the penis. His lesions had been previously treated several times with liquid nitrogen and electrodessication without causing any pigmentary changes, but his problem had relapsed half a month before presentation and now he was presenting with five new warts. The patient was treated with electrodessication to remove his condylomata acuminata. When his wound healed 12 days later, he was advised to use imiquimod $5 \%$ cream for relapse prevention. He applied the cream for three nights weekly and washed it off in the morning. Before long, he noticed 


\begin{tabular}{|c|c|c|c|c|c|c|c|}
\hline Case & Author (year) & $\begin{array}{l}\text { Age (year)/ } \\
\text { gender }\end{array}$ & Diagnosis & Site & Clinical presentation & Pathology & $\begin{array}{l}\text { Family } \\
\text { genetic } \\
\text { history }\end{array}$ \\
\hline 1 & Brown et al. 2005 [5] & $25 / M$ & $\begin{array}{l}\text { Vitiligo-like } \\
\text { hypopigmentation }\end{array}$ & Scrotum & $\begin{array}{l}\text { Multiple depigmented } \\
\text { patches on the scrotum } \\
\text { ranging from } 3 \mathrm{~mm} \text { to } 2 \mathrm{~cm}\end{array}$ & No & No report \\
\hline 2 & Stefanaki et al. 2006 [6] & $32 / \mathrm{M}$ & Vitiligo & $\begin{array}{l}\text { Dorsal surface of penis, } \\
\text { scrotum, and pubic area }\end{array}$ & Vitiligo, slight repigmentation & No & Yes \\
\hline 3 & Senel et al. 2007 [8] & $32 \mathrm{M}$ & $\begin{array}{l}\text { Vitiligo-like } \\
\text { depigmentation }\end{array}$ & $\begin{array}{l}\text { Glans penis, shaft of penis, } \\
\text { and scrotum }\end{array}$ & Depigmented areas & No & No \\
\hline 4 & Al-Dujaili et al. 2007 [7] & $21 / M$ & Vitiligo & Penile shaft and scrotum & Depigmented patches & No & No report \\
\hline 5 & Serrão et al. 2008 [9] & $26 / M$ & $\begin{array}{l}\text { Vitiligo-like } \\
\text { depigmentation }\end{array}$ & Shaft of penis & Vitiligo-like depigmentation & No & No report \\
\hline 6 & Zhang et al. 2011 [10] & $25 / M$ & Vitiligo & $\begin{array}{l}\text { Coronoid sulcus and } \\
\text { corpus penis }\end{array}$ & $\begin{array}{l}\text { Ivory-white patch nearly } \\
4 \times 2 \mathrm{~cm}\end{array}$ & No & No report \\
\hline 7 & Zhang et al. 2011 [10] & $22 / \mathrm{M}$ & Vitiligo & Penis & $\begin{array}{l}\text { Depigmented patches with } \\
\text { irregular pigmented edges }\end{array}$ & No & No report \\
\hline 8 & Wang et al. 2013 [11] & $36 / F$ & Vitiligo & Perineum and perianal & $\begin{array}{l}\text { Depigmentation patches } \\
\text { with clear demarcation lines }\end{array}$ & No & No \\
\hline
\end{tabular}

some irritation from erythema and excoriation in the treated areas, but he persisted in the application. After about 12 weeks of continuous use, he again presented to the clinic complaining of vitiligo-like depigmentation of the macules on his penis. He was instructed to stop using the imiquimod, but the macules in the treated areas gradually enlarged and asymptomatically merged; a vitiligo-like, depigmented patch also appeared on his scrotum during the following 10 days. After 4 weeks of applying tacrolimus $0.1 \%$ ointment, the lesions became slightly repigmented, and the area of vitiligo lesions stabilized. Unfortunately, no further repigmentation occurred after 3 months of follow-up.

Laboratory examination and a skin biopsy were recommended. He and his family members had no history of vitiligo, other depigmented dermatoses, or autoimmune disorders. He denied use of any other topical treatment. Physical examination showed vitiligo patches involving the glans penis, the shaft of the penis, and the scrotum, along with some remaining pigmented areas within the vitiligo plaques (Figure 1). Wood's light accentuated the depigmented areas. He was not tired or irritable. EKG, chest X-ray, and thyroid and abdominal ultrasonic scans indicated no cardiac, pulmonary, bilateral thyroid, hepatic, splenic, nephritic, or other organ involvement.

Additional laboratory analysis revealed normal counts of white blood cells, red blood cells and blood platelets; normal elevated erythrocyte sedimentation rate; normal thyroid function tests; negative antistreptolysin $\mathrm{O}$ titer and rheumatoid factor; normal serum immunoglobulins G, M, and A, E levels; normal liver enzyme level; normal blood urea nitrogen and creatinine level; normal blood glucose level; negative antinuclear and antineutrophil cytoplasmic antibodies; negative HIV and syphilis antibodies; normal hepatitis A, B, and C serology; normal urine and stool analysis.

A skin biopsy was performed on the dorsal surface of the penis, which showed a complete absence of melanocytes and melanin granules in the basal layer but with a normal dermis (Figure 2). Loss of melanocytes and melanin granules in the epidermis was highlighted by Fontana-Masson staining (Figure 3). Melanocytes and melanin granules exist in the epidermal basal-cell layer of normal skin (Figure 4). He was diagnosed, clinically and pathologically, with imiquimod-induced localized vitiligo.

\section{Discussion}

Imiquimod is an immune response modifier with the chemical structure 1-(2-methylpropyl)-1H-imidazo[4,5-c] quinolin-4-amin. Since imiquimod was authorized on the US market in 1997 and on the Chinese market in 2003, it has been approved as a patient-applied topical treatment for condylomata acuminata in adults. We find it is a welltolerated drug but with the frequent side effects of erythema, burning, blistering, and excoriation. We also noted its other adverse effect of inducing localized vitiligo, which occurred in the treated areas.

Vitiligo is a condition characterized by depigmentation of the skin and mucous membranes, with well-demarcated, depigmented macules and patches. The etiology of vitiligo is unknown, but research suggests that it may involve autoimmune, genetic, and other factors. Autoimmune disorders are often associated with thyroid abnormalities, pernicious anemia, systemic lupus erythematosus, and other diseases. Our patient denied any family history of vitiligo or 

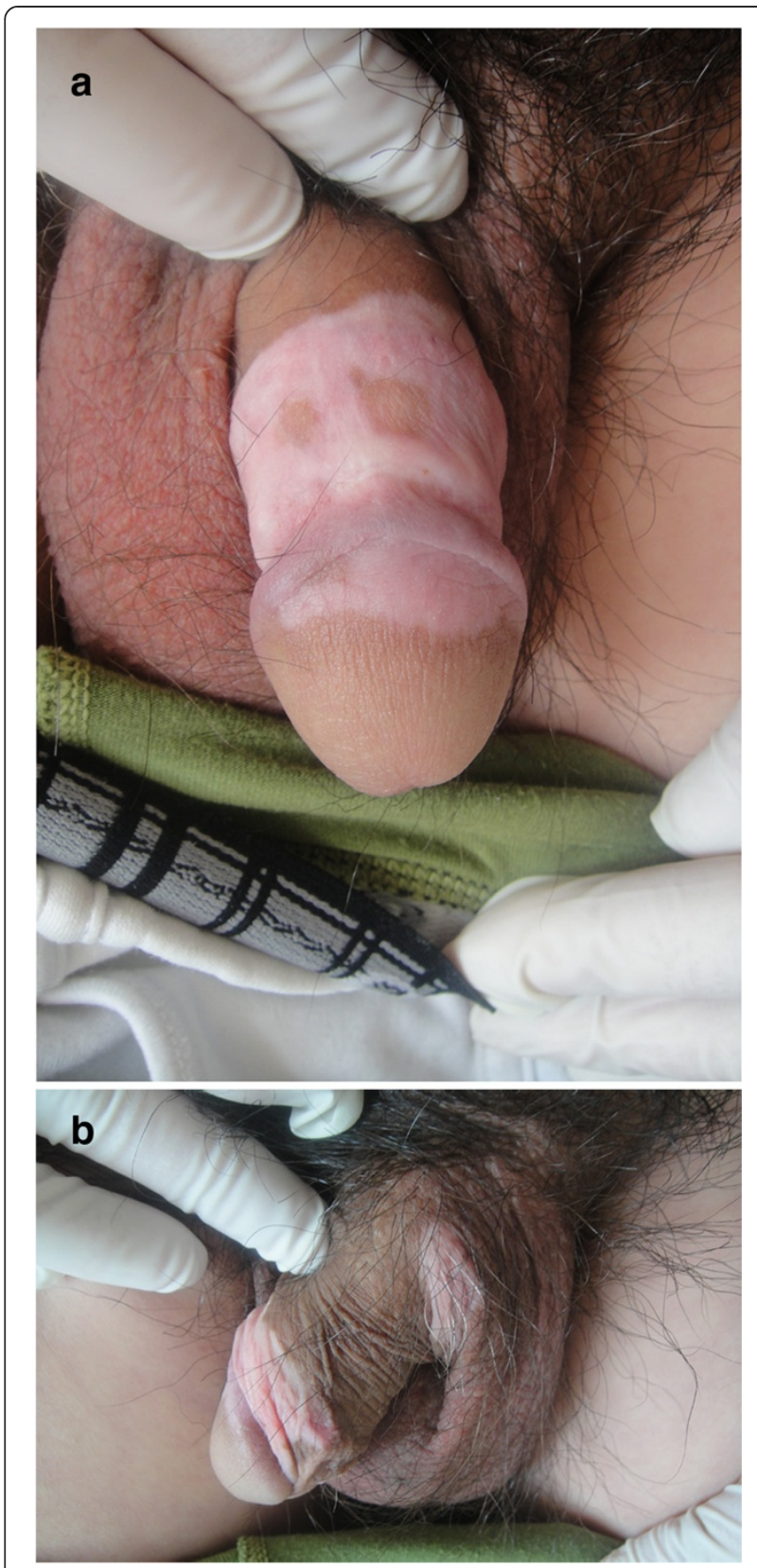

Figure 1 Vitiligo patches involving the glans penis, shaft of the penis, and scrotum after the use of imiquimod 5\%. a. Vitiligo on the patient's penis,some remaining pigmented areas within the vitiligo. plaques. b. vitiligo on the patient's scrotum.

autoimmune disorder, the possibility of which was excluded by laboratory analysis. He denied use of any other topical treatments in the areas treated with imiquimod 5\% cream. The features of his depigmented patches and histopathology support the diagnosis of vitiligo. Therefore, we believe that the vitiligo patches of our patient were induced by the imiquimod. We were able to obtain a small amount of superficial skin from the dorsal surface of the genital lesions in the perineal region. All eight of the patients

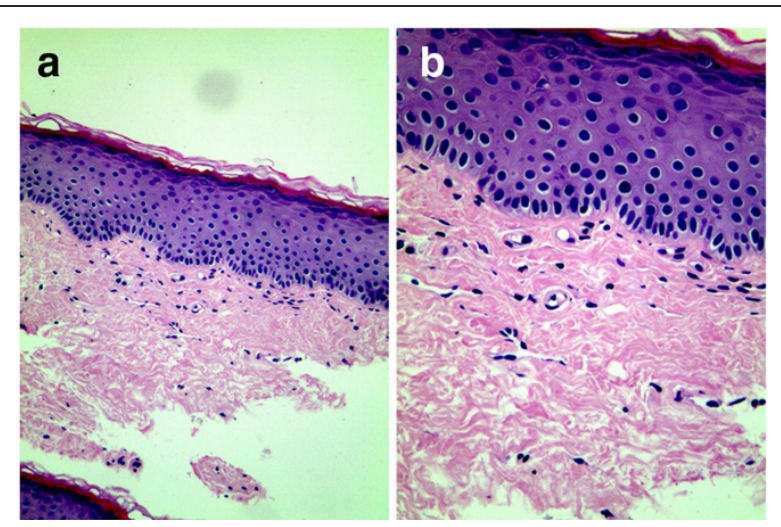

Figure $\mathbf{2}$ Loss of melanocytes and melanin granules. a. Normal stratum corneum, stratum granulosum, and stratum spinosum, with loss of melanocytes and melanin granules in the basal layer. Dermis showing no abnormalities $(H \& E \times 100)$. b. loss of melanocytes and melanin granules in the basal layer (H\&E $\times 200)$.

reported in the English literature refused biopsies of the depigmented areas, and one even refused to have the lesions photographed. Therefore, our patient is the first to undergo histopathological examination and whose diagnosis was based on clinical and histopathological findings.

The possible mechanism for the destruction of the human papilloma virus (HPV) by imiquimod is that it stimulates peripheral blood monocytes, macrophages, and dendritic cells to produce such cytokines as interferon alfa (IFN- $\alpha$ ), interleukin-12 (IL-12), and tumor necrosis factor alfa (TNF- $\alpha$ ), so imiquimod can enhance the host's innate and cellular immune response and combat anogenital HPV infection [12,13]. To study the safety and effectiveness of imiquimod $5 \%$ cream in the treatment of external anogenital warts, Edwards et al. applied it on 109 patients; 50\% of the patients experienced eradication of all treated baseline warts [14].

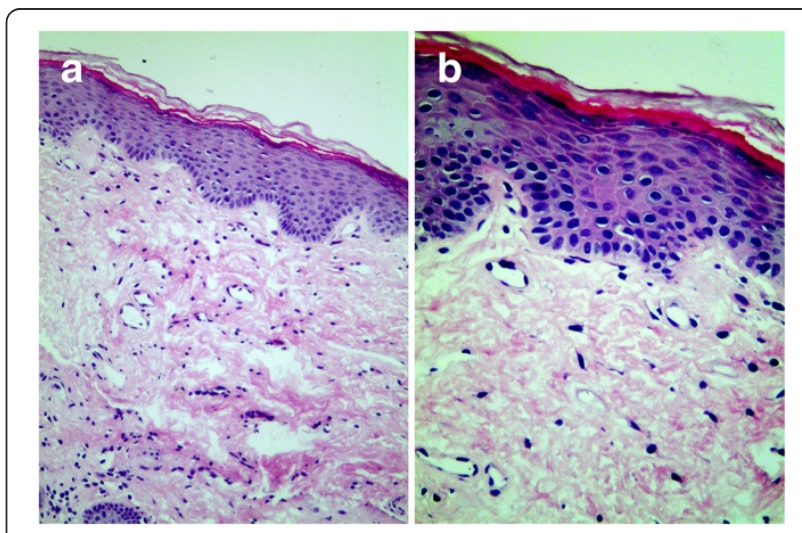

Figure 3 Absence of melanin granules. a. Absence of melanin granules in epidermis. Normal dermis. (Masson-Fontana stain for melanin $\times 100$ ). $\mathbf{b}$. Absence of melanin granules in epidermal basal cell layer. (Masson-Fontana stain for melanin $\times 200$ ). 


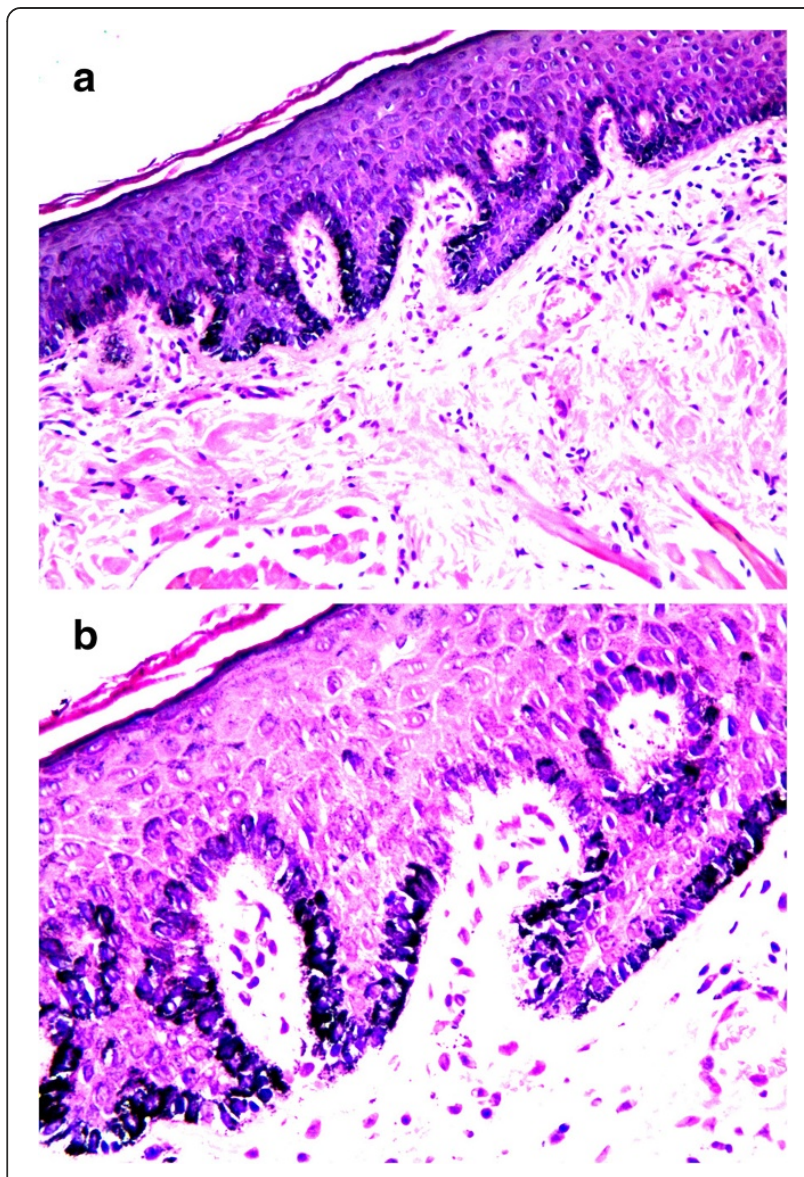

Figure 4 Normal skin (control): normal of melanocytes and melanin granules. a. presence of melanocytes and melanin granules in epidermis. Normal dermis (Masson-Fontana stain for melanin $\times 100$ ). b. presence of melanocytes and melanin granules in epidermal basal cell layer. (Masson-Fontana stain for melanin $\times 200$ ).

Other studies also indicated that imiquimod 5\% cream was effective in treating condylomata acuminata. However, imiquimod not only kills the HPV but also destroys melanocytes. Similarly, the mechanism of imiquimodinduced vitiligo may be that the medication activates the Langerhans cells in the lesions via antigen presentation, leading to the destruction and apoptosis of the melanocytes. Imiquimod-induced apoptosis of melanocytes was confirmed by TUNEL assay, Hoechst 33258 staining, and measuring mitochondrial membrane potential in melanocytes [15]. Moreover, imiquimod can induce cytokines such as IFN- $\alpha$, TNF- $\alpha$, IL-6, IL-8, and nitric oxide to cause vitiligo [16]. Additionally, imiquimod binds to Toll-like receptor-7 and -8 , increasing production of proinflammatory cytokines such as IFN- $\alpha$, TNF- $\alpha$, and LI-12 [17,18], which play a role in the pathogenesis of vitiligo.

Accompanying the use of imiquimod on increasing numbers of patients with condylomata acuminata, dermatologists should keep this potential side effect in mind.

\section{Conclusion}

Imiquimod 5\% cream, as an immune response modifier and a safe drug, is used to treat condylomata acuminata, basal cell carcinoma, Bowen's disease, common and plantar warts, molluscum contagiosum, and other disorders. However, mild-to-moderate, local and systemic, adverse effects of imiquimod may occasionally occur. Among its adverse effects, imiquimod-induced vitiligo should be anticipated when dermatologists prescribe this drug.

\section{Consent}

Written informed consent was obtained from the patient for publication of this case report and any accompanying images. A copy of the written consent is available for review by the Editor of this journal. This study was proved by Institutional Review Board of Qianfoshan Hospital, Shandong University.

\section{Competing interests}

The authors declare that they have no potential conflicts of interest to disclose.

\section{Authors' contributions}

Study concept and design: WFL. Acquisition of data: HYX and LZG. Analysis and interpretation of data: HYS and WC. Drafting of the manuscript: HYX and LZG. Critical revision of the manuscript for important intellectual content: WFL. All authors read and approved the final manuscript.

\section{Acknowledgments}

We would like to thank Dr.Qing Sun, Dr.Ping Zhou, Dr.Wei Lu in qianfoshan hospital, Shandong university for helping in histological analysis. We also thank Dr. Xianmei Lu in Shandong provincial skin Hospital, Shandong University. This study was supported by the grant from Natural Science Foundation of Shandong (No. Y2008C160).

\section{Author details}

'Department of Dermatology, Qianfoshan Hospital, Shandong University, 16766\# Jing-Shi Road, Jinan 250014, China. ${ }^{2}$ Shandong Provincial Chest Hospital, Jinan 250013, China. ${ }^{3}$ Taishan Medical college, Tai'an 271000, China. ${ }^{4}$ Department of Dermatology, Jinan city central Hospital, Jinan 250013, China. ${ }^{5}$ Department of Dermatology, Jinan Sixth People's Hospital, Jinan 250200, China.

Received: 12 March 2014 Accepted: 6 June 2014

Published: 13 June 2014

\section{References}

1. Castle PE, Gage JC, Partridge EE, Rausa A, Gravitt PE, Scarinci IC: Human papillomavirus genotypes detected in clinician-collected and selfcollected specimens from women living in the Mississippi Delta. BMC Infect Dis 2013, 13:5.

2. Shi H, Zhang X, Ma C, Yu N, Wang J, Xia L, Ge X, Liu M, Duan A: Clinical analysis of five methods used to treat condylomata acuminata. Dermatology 2013, 227:338-345.

3. Chen FP: Efficacy of imiquimod $5 \%$ cream for persistent human papillomavirus in genital intraepithelial neoplasm. Taiwan J Obstet Gynecol 2013, 52:475-478.

4. Rosenblatt A, de Campos Guidi HG: Local and systemic adverse effects of imiquimod therapy for external ano condyloma acuminatum in men: report of three cases. Int J STD AIDS 2012, 23:909-910.

5. Brown T, Zirvi M, Cotsarelis G, Gelfand JM: Vitiligo-like hypopigmentation associated with imiquimod treatment of condyloma acuminatum. J Am Acad Dermatol 2005, 52:715-716.

6. Stefanaki C, Nicolaidou E, Hadjivassiliou M, Antoniou C, Katsambas A: Imiquimod-induced vitiligo in a patient with condyloma acuminatum. $J$ Eur Acad Dermatol Venereol 2006, 20:755-756. 
7. Al-Dujaili Z, Hsu S: Imiquimod-induced vitiligo. Dermatol Online J 2007, 13:10.

8. Senel E, Seckin D: Imiquimod-induced vitiligo-like depigmentation. Indian J Dermatol Venereol Leprol 2007, 73:423.

9. Serrão W, Páris $F R$, Feio $A B$ : Genital vitiligo-like depigmentation following use of imiquimod 5\% cream. Eur J Dermatol 2008, 18:342-343.

10. Zhang R, Zhu W: Genital vitiligo following use of imiquimod $5 \%$ cream. Indian J Dermatol 2011, 56:335-336.

11. Wang HW, Miao F, Shi L, Lü T, Huang Z, Wang XL: Imiquimod-induced localized vitiligo in wife and lichen planus in husband. Chin Med J (Engl) 2013, 126:2593.

12. Schöfer H, Van Ophoven A, Henke U, Lenz T, Eul A: Randomized, comparative trial on the sustained efficacy of topical imiquimod $5 \%$ cream versus conventional ablative methods in external ano condyloma acuminatum. Eur J Dermatol 2006, 16:642-648,

13. Skinner RB Jr: Imiquimod. Dermatol Clin 2003, 21:291-300. Review.

14. Edwards L, Ferenczy A, Eron L, Baker D, Owens ML, Fox TL, Hougham AJ, Schmitt KA: Self-administered topical 5\% imiquimod cream for external anogenital warts: HPV Study Group. Human PapillomaVirus. Arch Dermatol 1998, 134:25-30.

15. Kim CH, Ahn JH, Kang SU, Hwang HS, Lee MH, Pyun JH, Kang HY: Imiquimod induces apoptosis of human melanocytes. Arch Dermatol Res 2010, 302(4):301-306. doi:10.1007/s00403-009-1012-0. Epub 2009 Dec 23.

16. Ueyama A, Yamamoto M, Tsujii K, Furue Y, Imura C, Shichijo M, Yasui K: Mechanism of pathogenesis of imiquimod-induced skin inflammation in the mouse: a role for interferon-alpha in dendritic cell activation by imiquimod. J Dermatol 2014, 41:135-143.

17. Schön MP, Schön M: Imiquimod: mode of action. Br J Dermatol 2007, 157(Suppl 2):8-13.

18. Lacarrubba F, Nasca MR, Micali G: Advances in the use of topical imiquimod to treat dermatologic disorders. Ther Clin Risk Manag 2008, 4:87-97.

doi:10.1186/1471-2334-14-329

Cite this article as: Li et al:: Induction of vitiligo after imiquimod treatment of condylomata acuminata. BMC Infectious Diseases 2014 14:329.

\section{Submit your next manuscript to BioMed Central and take full advantage of:}

- Convenient online submission

- Thorough peer review

- No space constraints or color figure charges

- Immediate publication on acceptance

- Inclusion in PubMed, CAS, Scopus and Google Scholar

- Research which is freely available for redistribution 